\title{
Jovicentric latitude effect on the bKOM radio emission observed by Ulysses/URAP
}

\author{
C. H. Barrow ${ }^{1}$, A. Lecacheux ${ }^{2}$, and R. J. MacDowall ${ }^{3}$ \\ 1 Max-Planck-Institut für Aeronomie, 37189 Katlenburg-Lindau, Germany \\ 2 ARPEGES, Observatoire de Paris, 92195 Meudon, France \\ 3 NASA Goddard Space Flight Center, Greenbelt, MD 20771, USA
}

Received 29 August 2000 / Accepted 3 November 2000

\begin{abstract}
During 1994 and into 1996, Ulysses was at distances of 5 AU or more from Jupiter and travelling from south to north of the ecliptic plane. At these distances, the jovian broadband radio emission (bKOM) was only received occasionally by the Unified Radio and Plasma Experiment (URAP) on board the Ulysses spacecraft. The signals were generally weak and much care is needed to find and to identify the events. It is found that the visibility and polarization of the bKOM appears to depend upon the jovicentric latitude of Ulysses $\left(D_{\text {Uly }}\right)$ at the time of observation. All but two of the bKOM events observed when Ulysses was at northern jovicentric latitudes were predominantly right-hand $(\mathrm{RH})$ polarized while events recorded when the spacecraft was at southerly jovicentric latitudes were all predominantly LH polarized, the change taking place somewhere between $3.9^{\circ} \leq D_{\mathrm{Uly}} \leq 8.8^{\circ}$, close to $0^{\circ}$ jovimagnetic latitude. Compared with previous observations of the bKOM, made by spacecraft considerably closer to Jupiter, the present occurrence probabilities were lower for the LH polarized events although the distribution was similar. For the RH polarized events, however, the distribution was different, the so-called main peak being absent or, perhaps, displaced towards a larger central meridian longitude. It is shown that, in a two-dimensional model, if cyclotron maser emission in a dipole magnetic field is assumed for Jupiter, the detection of bKOM at a given frequency by a spacecraft at a specified location, determines a unique value of $\beta$ for an assumed value of $L$ and a given field model. This is not true for a three-dimensional model. The present results raise questions on the application of the cyclotron maser theory to bKOM emission.
\end{abstract}

Key words. space vehicles - planets and satellites: Jupiter - radio continuum: solar system

\section{Introduction}

Observations by the Radio Astronomy Explorer (RAE-1) and the Interplanetary Monitoring Platform 6 (IMP 6) gave the first indications of jovian radio emission at frequencies close to $1 \mathrm{MHz}$ (Brown 1974; Desch \& Carr 1974; Kaiser 1977). The Voyager Planetary Radio Astronomy (PRA) experiment established the existence of four components in the low-frequency radio spectrum of Jupiter; three components were distinguished at frequencies below the critical frequency of Earth's ionosphere, in addition to the decametre-wave radiation (DAM), already well-known from over thirty years of systematic ground-based observations. The low-frequency components were classified as a hectometre-wave component (HOM) and two kilometrewave components, a narrow-band emission (nKOM) and a broad-band emission (bKOM). The characteristics of each of these components have been reviewed by Alexander et al. (1981), Boischot et al. (1981), Carr et al. (1983),

Send offprint requests to: C. H. Barrow,

e-mail: barrow@linmpi.mpg.de
Kaiser \& Desch (1984), Leblanc \& Daigne (1985), Boischot (1988) and Leblanc (1988).

The present paper is concerned with observations of the bKOM made by the Ulysses Unified Radio and Plasma (URAP) experiment (Stone et al. 1992a). In a previous study, reported briefly at the Magnetospheres of the Outer Planets conference in August, 1999, (Barrow et al. 2001) there were indications of the influence of the jovicentric latitude of Ulysses on observations of the visibility and polarization of the bKOM, beyond the inferences that could be made from observations close to encounter in 1992 . This effect has now been studied further and is reported here in greater detail.

\section{Ulysses antennas and receivers}

The receivers cover two bands, from 1.25 to $48.5 \mathrm{kHz}$ (loband) and from 52 to $940 \mathrm{kHz}$ (hi-band). Hi-band operates in 12 channels, approximately logarithmically spaced, each frequency being determined by one of twelve crystal local oscillators. The intermediate frequency (IF) amplifier 


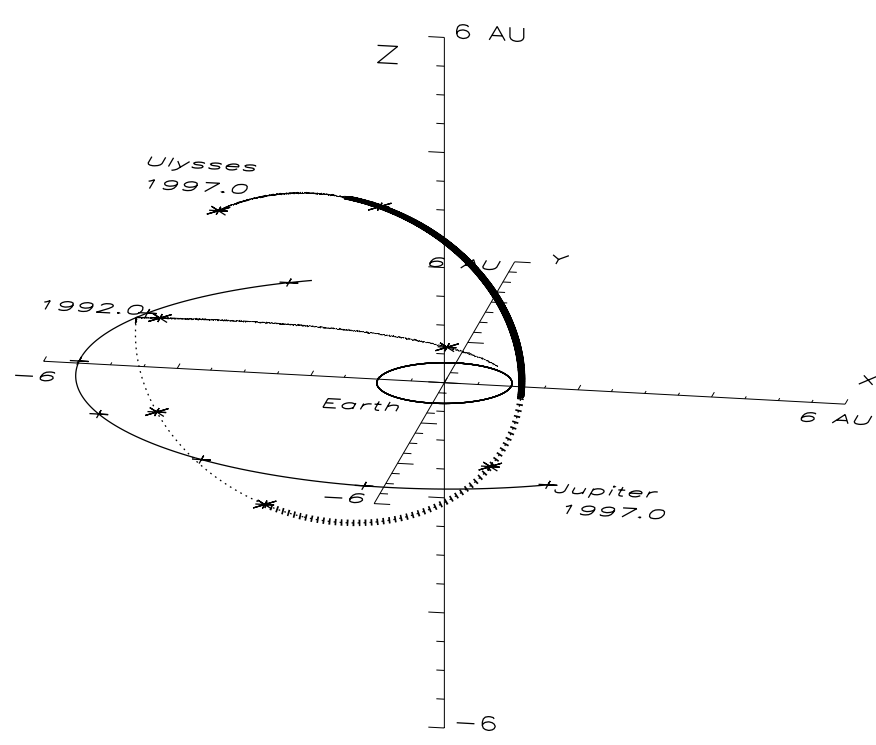

Fig. 1. Trajectories of Ulysses and Jupiter. The heavy line represents the Ulysses' trajectory when the observations were made

frequency is $10.7 \mathrm{MHz}$, the dynamic range about $70 \mathrm{~dB}$ and the bandwidth $3 \mathrm{kHz}$. Lo-band operates in 64 channels, arithmetically spaced at $0.75 \mathrm{kHz}$ intervals. The IF amplifier frequency is $432.25 \mathrm{kHz}$, the dynamic range about $70 \mathrm{~dB}$ and the bandwidth $750 \mathrm{~Hz}$. The receivers are connected to a $72 \mathrm{~m}$ wire antenna perpendicular to the spacecraft spin axis and to a $7.5 \mathrm{~m}$ monopole antenna along the spin axis. The spacecraft and the antenna system spin with a $12 \mathrm{~s}$ period. The inputs from the antennas can be combined to synthesize an equivalent dipole tilted with respect to the spin axis. By combining the inputs with suitable phase differences the polarization of the incoming waves can be determined (Manning \& Fainberg 1980; Stone et al. 1992b). Polarization can only be calculated from hi-band data. The sensitivity, when used in the separation mode (Stone et al. 1992a), is about $S_{\min } \simeq 510^{4} \mathrm{Jy}$ at $100 \mathrm{kHz}$. In the summation mode (SUM), for polarization measurements, the sensitivity is down by about $10 \mathrm{~dB}$. Polarization measurements of the radiation have been used to improve existing knowledge of the source location and the beaming characteristics of the bKOM.

\section{Observations}

URAP observations were examined for the period January 1, 1994, to March 1, 1996, (940101 to 960301, a total of 790 days). During this period, Ulysses was at distances of about 4.7 to $7.2 \mathrm{AU}$ from Jupiter and travelling from south to north of the ecliptic plane (Fig. 1), the jovicentric latitude of the spacecraft changing from about $-36^{\circ}$ to $20^{\circ}$. The bKOM was only received occasionally and the number of events suitable for study was further limited by the considerations outlined in the following two paragraphs.

At distances greater than $5 \mathrm{AU}$, the problem of finding and identifying the bKOM requires much care. No formal listing of the jovian events observed exists for the spacecraft. At these distances the bKOM emission is generally weak and often lost in solar emission, auroral kilometric radiation (AKR) and background noise. Events cannot be identified in a sequence of spectra for several days. Each day has to be searched carefully, the sensitivity of the spectra needs to be enhanced and the background may need to be changed. Identification is by the characteristic spectral form and polarization of the emission as well as the appearance in simultaneous time series at several different frequencies where non-characteristic frequency drifts can easily be recognized. For example, the drift and the absence of strong polarization of a weak solar type III burst will distinguish it from a weak bKOM event. The selection of events for study is further limited by the need for polarization data which is only obtainable when the receiver is operating in SUM mode (Stone et al. 1992a). Only 57 events, recorded over 51 days, eventually met these criteria.

We also recall that, immediately after Jupiter encounter in 1992, when Ulysses was at an extreme southerly jovicentric latitude $\left(D_{\mathrm{Uly}} \simeq-38^{\circ}\right)$, events showing some similarity to both the $\mathrm{HOM}$ and the bKOM were found to occur at different frequencies to those typical of previous observations by Voyager (Barrow \& Lecacheux 1995). These emissions, arbitrarily called HOMa and bKOMa, may also be present as another confusing feature of the spectra studied here as well as the "unidentified emission" reported in the same paper. It is possible, therefore, that some very weak bKOM events may have occurred and passed unnoticed.

Only bKOM events which could be identified with a good degree of certainty and for which polarization data are available have been considered. Event occurrence times could be measured from the spectra or from single frequency intensity-time plots. The maximum estimated uncertainty was about $\pm 5 \mathrm{~min}$ or $\pm 3^{\circ}$ of CML. System III Central Meridian Longitudes (CML) for Ulysses are taken from the NASA SEDR file and corrected for the light-travel time from Jupiter to the spacecraft.

Typical spectra, taken on 950611 (DOY 94527) when Ulysses was at jovicentric latitude $D_{\text {Uly }}=11.6^{\circ}$, are shown in Figs. 2 and 3. In Fig. 2, both the URAP hiband and lo-band spectra are shown. These spectra were used to find and to identify the bKOM events. The time resolution of both hi- and lo-band data sets is $144 \mathrm{~s}$. For the lo-band spectrum, three adjacent frequencies are averaged to remove most of the modulation imposed due to the spinning spacecraft-antenna system. Three frequencies are collected in six seconds, which is approximately onehalf of the spacecraft spin period. In hi-band, the data at each frequency are collected for $12 \mathrm{~s}$; these are averaged together and so spin-modulation should not be present in these data. Note that much of the typical structure of the bKOM has been smoothed out by the effects of temporal broadening due to scattering over the distance travelled by the radiation through the interplanetary medium (Barrow et al. 1999).

The polarization is represented as a spectrum of the degree of circular polarization $m_{\mathrm{c}}$ shown in the second 

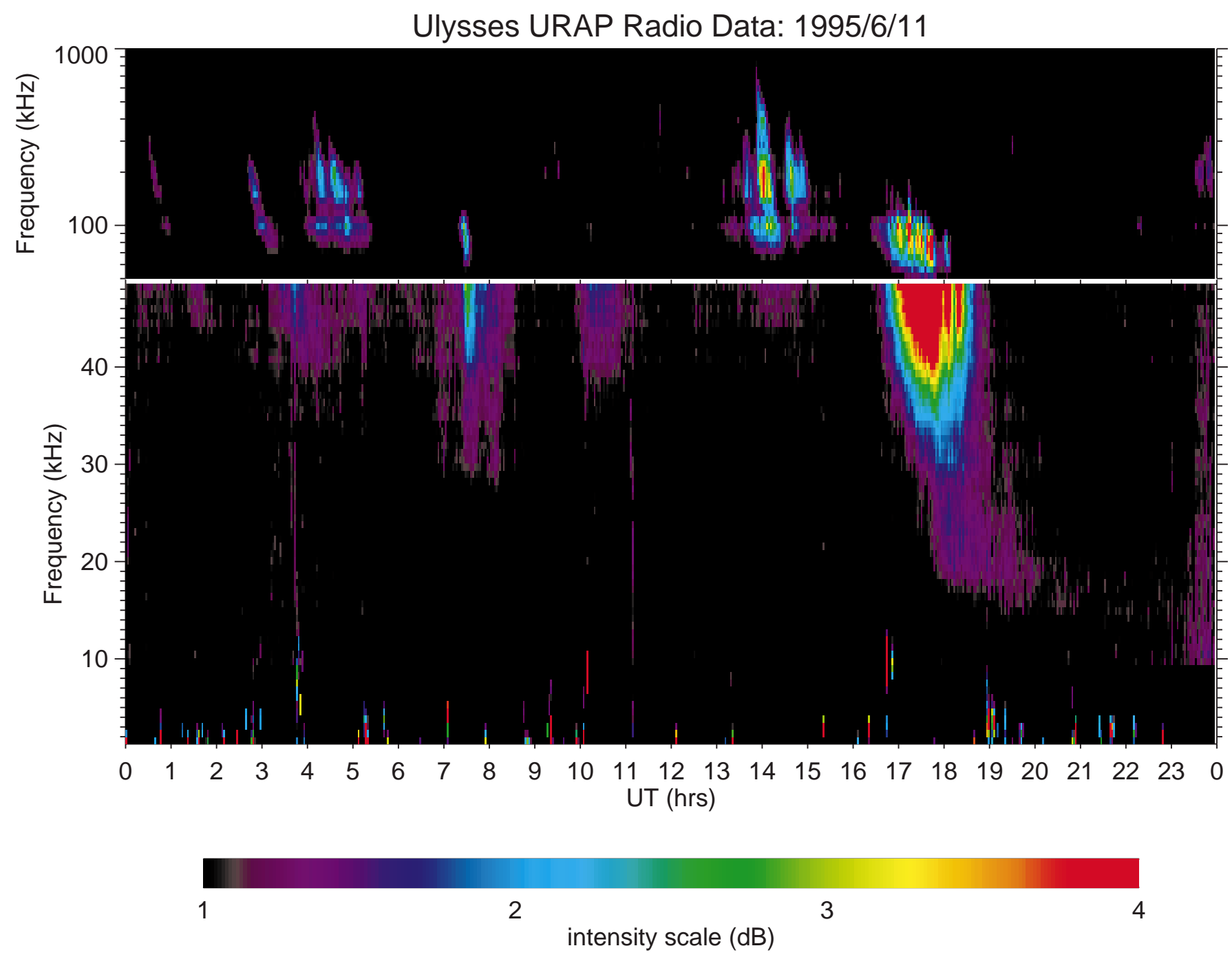

Fig. 2. Hi- and lo-band Ulysses/URAP spectra for 950611 (DOY 94527)

panel of Fig. 3. The total intensity $I_{\mathrm{L}}+I_{\mathrm{R}}$ measured by URAP is presented in the top panel. Then $m_{\mathrm{c}}$, is given by

$m_{\mathrm{c}}=\frac{I_{\mathrm{L}}-I_{\mathrm{R}}}{I_{\mathrm{L}}+I_{\mathrm{R}}}$

where $I_{\mathrm{L}}$ and $I_{\mathrm{R}}$ are, respectively, the $\mathrm{LH}$ and RH polarized intensities. This could only be calculated for hi-band data and so the predominant polarization in the lowest hiband frequency of $52 \mathrm{kHz}$ was taken as the predominant polarization of the event.

In the third panel, the azimuth, with respect to the direction of the Sun, gave an additional identification criterion and, in particular, distinguished jovian emission from possible saturnian (SKR) emission (Lecacheux \& Aubier 1997). The position of each planet is indicated by the letters " $J$ " or " $S$ " adjacent to the colour scale. The modulation index, shown in the fourth panel, is essentially an indication of the certainty of identification. The numbers above the top panel represent the rotation number of Jupiter, taken as zero on January 1, 1982, and the CML, corrected for light-travel time from Jupiter to Ulysses.
It can be seen that a bKOM event was observed by Ulysses to begin close to $1630 \mathrm{UT}\left(\mathrm{CML} \simeq 273^{\circ}\right)$. A weaker event occurred at about 0730 UT. Both of these events were $\mathrm{RH}$ polarized (i.e. $m_{\mathrm{c}}<1$; the emission originated in the northern hemisphere of Jupiter) and were separated by a time interval roughly equal to the rotation period of the planet, a little less than 10h. Two other periods of $\mathrm{RH}$ polarized activity, which may have been bKOMa emission, can be seen at about 0400 and 1400 UT. These two events are also separated by a time interval close to the rotation period of the planet. The azimuth spectrum shows that all of these emissions originated from the direction of Jupiter.

Note that the interplanetary medium will cause scattering effects in the emission (Barrow et al. 1999). Faraday rotation will tend to depolarize linearly polarized radiation but it will only cause phase differences in circularly polarized radiation. Thus the actual sense of polarization will not be changed by scattering (Woan 1997, 1999).

All of the days when bKOM events were observed by Ulysses are shown in Fig. 4a, where it can be seen that, 

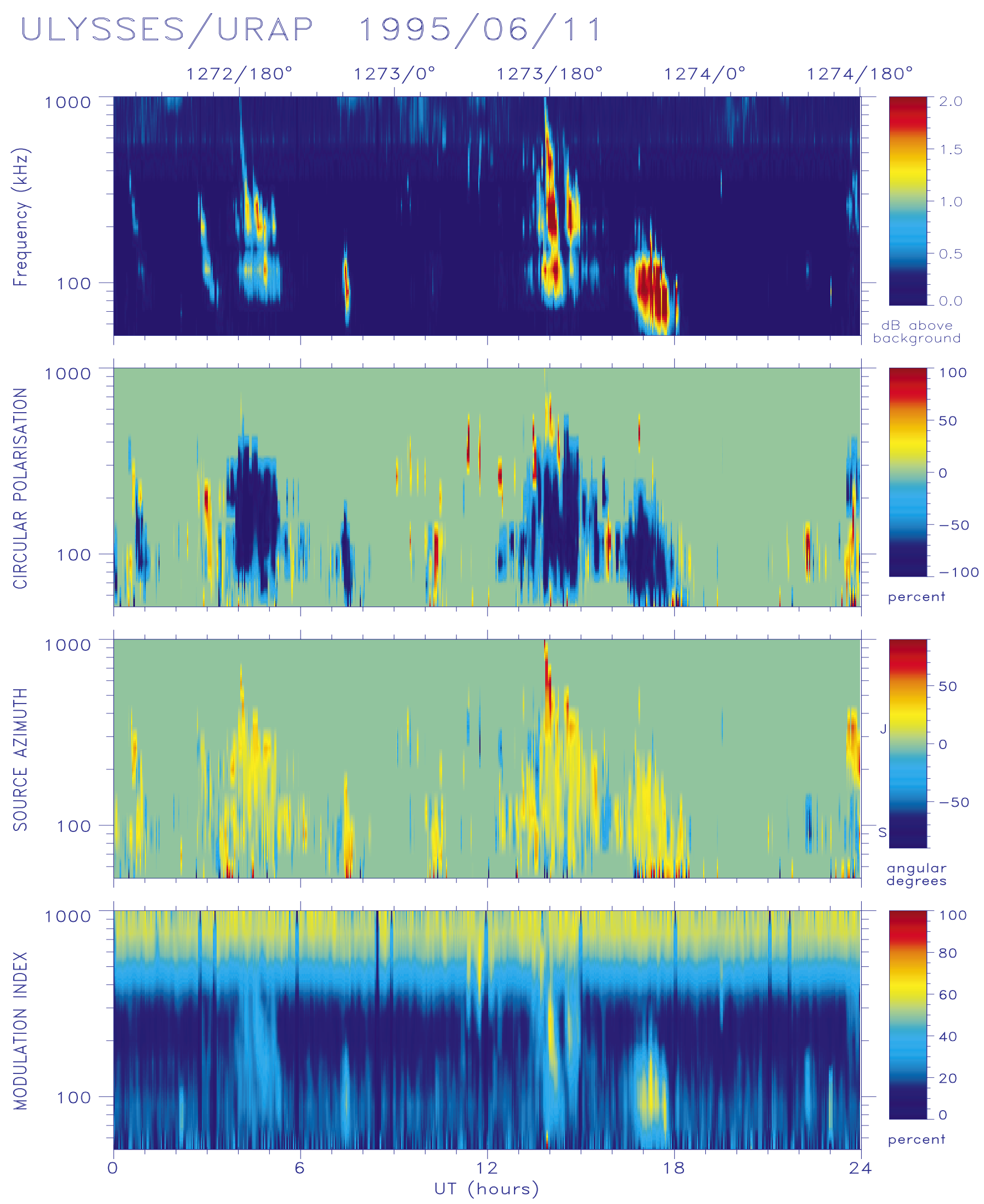

Fig. 3. Hi-band Ulysses/URAP spectra for 950611 (DOY 94527). The four panels, from top to bottom, show spectra of total intensity, degree of polarization, azimuth of the direction of origin of the emission with respect to the direction of the Sun, and modulation index. The numbers along the top of the first spectrum give the jovian rotation number (zero on 820101) and CML values, corrected for the light-travel time from Jupiter to the spacecraft. All of the jovian emission is RH polarized, notably the bKOM event beginning close to $1630 \mathrm{UT}$ 


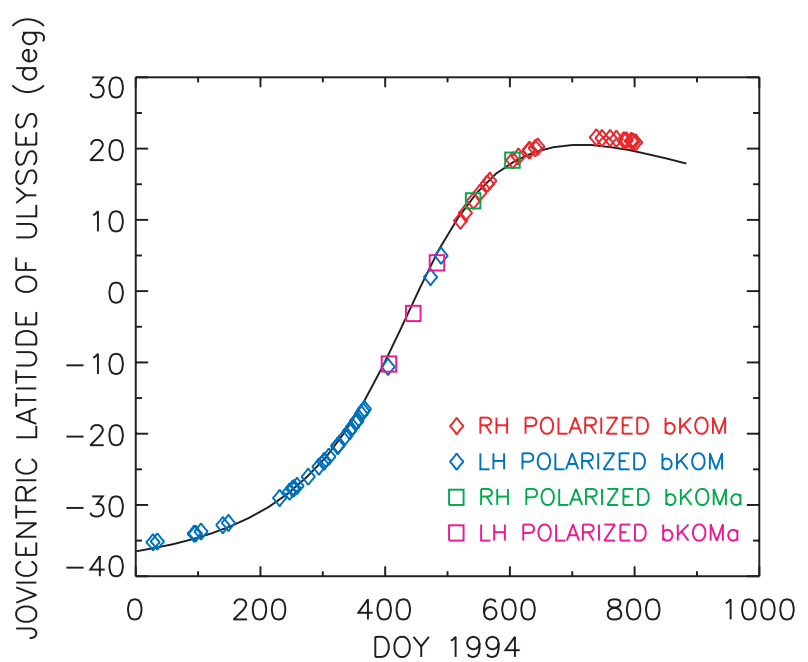

(a)

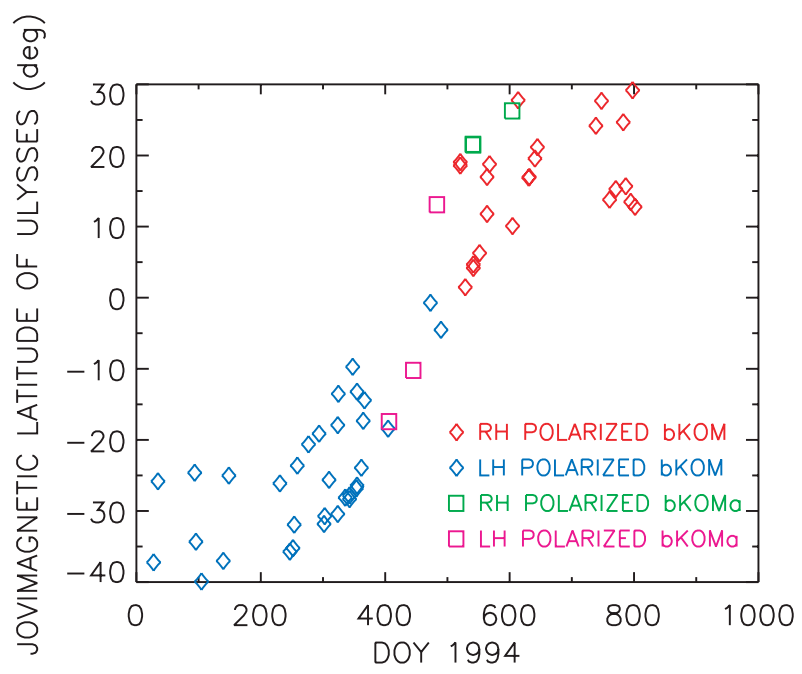

(b)

Fig. 4. Days on which bKOM events were observed by Ulysses, during the period 940101 to 960301 (DOY 94001 to 94790), against a) jovicentric latitude and $\mathbf{b}$ ) jovimagnetic latitude of the spacecraft

during the period studied (940101 to 960301), the RH polarized bKOM was only observed when the spacecraft was in northerly jovicentric latitudes while, with the exception of two events, LH polarized bKOM was only observed when the spacecraft was in southerly jovicentric latitudes. The two exceptional events both occurred close to crossing of the jovicentric equatorial plane and in both cases the jovimagnetic latitude was southerly. Several possible bKOMa events were also recorded. In Fig. 4b, the same days are plotted against the jovimagnetic latitude of Ulysses at the nearest hour to the event time and these, of course, show a spread of some $20^{\circ}$ corresponding to the $\pm 10^{\circ}$ tilt of the jovian magnetic field as the planet rotates.

There was no event recorded during the 31 days between 950420 and 950521 when the change in polarization from LH to RH took place and so we can only say that this

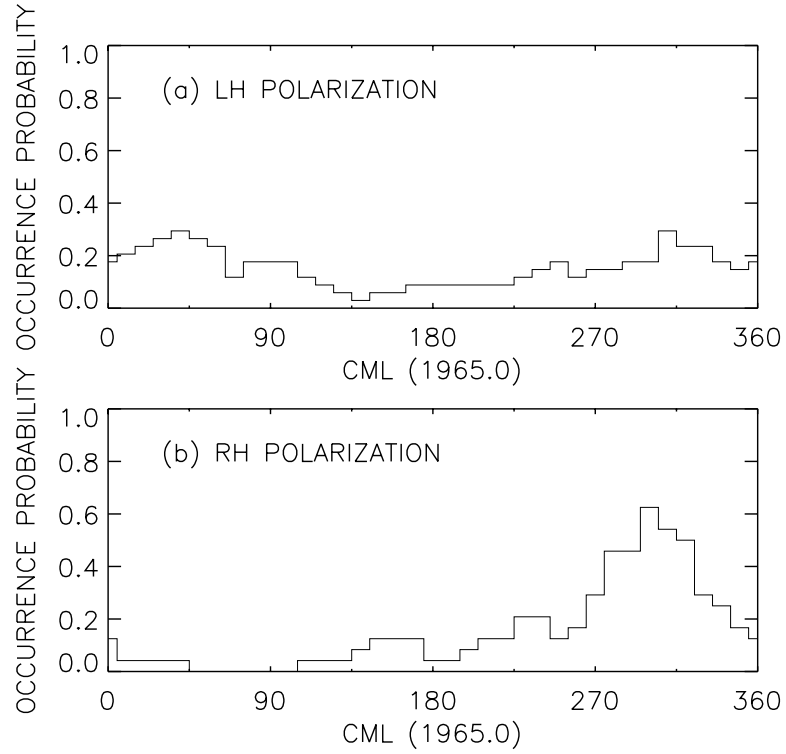

Fig. 5. Normalised occurrence probability of the bKOM for a) $\mathrm{LH}$ polarization, and b) $\mathrm{RH}$ polarization

occurred somewhere between $3.9^{\circ} \leq D_{\text {Uly }} \leq 8.8^{\circ}$. The corresponding jovimagnetic latitudes were, respectively, $-5.6^{\circ}$ and $18.0^{\circ}$. Figure $4 \mathrm{~b}$ shows that the changeover corresponds to the change in jovimagnetic latitude of Ulysses from south to north. The absence of activity during this period may be an effect of the jovicentric latitude of Ulysses but it could also be due to the increased difficulty of identification of the bKOM while the spacecraft is relatively close to the Sun.

Normalised occurrence probabilities for $\mathrm{LH}$ and $\mathrm{RH}$ polarized emission are shown in Figs. 5a and 5b, respectively. It can be seen that, although the occurrence probabilities are lower, the LH polarized distribution is essentially the same as that found previously at $52 \mathrm{kHz}$ (Barrow \& Lecacheux 1995). However, the RH polarized distribution differs in that the so-called main source peak, found in Voyager observations by Leblanc \& Daigne (1985), is either absent or, perhaps, displaced towards a larger CML.

\section{Discussion}

There have been a number of suggestions regarding the bKOM emission process (for example, Jones 1988; Leblanc 1988). More recently, there has been evidence that the bKOM, like the HOM, may be due to the CyclotronMaser instability (CMI) with dominant emission in the $\mathrm{R}-\mathrm{X}$ mode and source regions on L-shells between 9 and 15 (Ladreiter et al. 1994 and the references therein). The emission is beamed into a radiation pattern in the form of a thin-walled hollow cone with apex at the source and axis tangent to the magnetic field direction. Propagation is in the $\mathrm{R}-\mathrm{X}$ mode at frequencies equal to or just above the local gyrofrequency $f_{\mathrm{c}}$. An event is seen as continuous emission due to radiation from the edges of a succession of CMI cones, distributed over a range of CMLs, and rotating with the planet. In the discussion that follows we 
will assume that the bKOM is generated from tilted dipole field lines of the order of $10 \leq L \leq 15$.

Beaming can be represented by the two-dimensional geometry of the electron cyclotron frequency surface superimposed upon a dipolar jovian magnetic field, as sketched in Fig. 6, where the point $\mathrm{S}$ represents a bKOM source (i.e. the apex of an emission cone) in the southern magnetic hemisphere and the northern edge of the cone is directed towards the spacecraft when it is in a northerly magnetic latitude. If the gyrofrequency at $\mathrm{S}$ is $f_{\mathrm{c}}$ in $\mathrm{MHz}$ and the magnetic field $B$ is in Gauss, the magnetic field geometry is given by Eqs. (3) and (4), following Chapman \& Bartels (1951), and $L$ is defined by Eq. (5). It is evident that Eq. (6) follows from Fig. 6. Then we have,

$$
\begin{aligned}
f_{\mathrm{c}} & =2.8 B \\
r^{3} & =\frac{M}{B} \sqrt{3 \sin ^{2} \phi+1} \\
\tan \alpha & =0.5 \cot \phi \\
L & =\frac{R_{\mathrm{J}}}{\cos ^{2} \Lambda}=\frac{r}{\cos ^{2} \phi} \\
\beta & =\phi-\alpha \pm \delta
\end{aligned}
$$

where $M=4.28 \mathrm{GR}_{\mathrm{J}}^{3}$ is the magnetic moment of Jupiter, $\delta$ is the magnetic latitude of the spacecraft, $\beta$ is the emission cone half-angle, $\phi$ is the magnetic latitude of the source $\mathrm{S}$, $\alpha$ is the angle between the field line and OS at the point $\mathrm{S}, r$ is the distance OS of the source from the centre of Jupiter in $R_{\mathrm{J}}, L$ specifies the corresponding L-shell and $\Lambda$ is the invariant latitude of $L$. The geometry is the same for both hemispheres but, in Eq. (6), the positive/negative sign of $\delta$ refers to the spacecraft on the opposite/same side of the magnetic equator as the source.

It follows, from Eq. (6) that, in the two-dimensional model, the detection of bKOM at a given frequency $f_{\mathrm{c}}$ by a spacecraft at a given location specified by $\delta$, determines a unique value of $\beta$ for an assumed value of $L$ and a given field model. This is not true for the three-dimensional model.

It should be noted that the durations of most of the bKOM events considered here and shown in Fig. 4a, were relatively short; generally a little over an hour and considerably less than typical durations observed closer to the planet (see, for example, Barrow \& Lecacheux 1995; their Fig. 3). As Jupiter rotates, an observer sees the event as emission from a succession of CMI cones distributed over a range of CMLs. As the cone half-angles have, in general, been found by various workers to be large, estimates ranging from $30^{\circ}$ to $90^{\circ}$ (Ladreiter et al. 1994) and, as Jupiter rotates at a rate of about $36^{\circ} / \mathrm{hr}$, an event might be expected to last for some two or three hours as seen in observations close to Jupiter. It seems likely, therefore, that the shorter duration events seen at these greater distances from the planet and presented here may be due to emission from sectors of the extreme northern or southern edges of the CMI cones.

Also, if the source is on $L=15$, where this cuts the $f_{\mathrm{c}}=52 \mathrm{kHz}$ surface, the source will be at a jovimagnetic

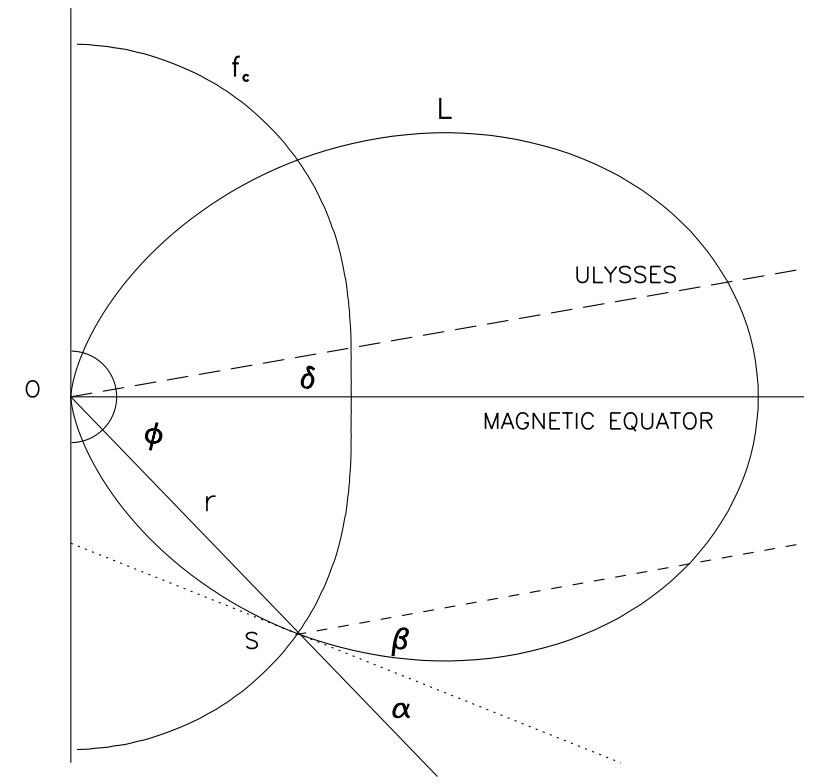

Fig. 6. Two dimensional beaming geometry for bKOM emission in a dipole magnetic field from a source on L-shell $\simeq 15$ close to the $f_{\mathrm{c}}=52 \mathrm{kHz}$ surface

latitude of about $45^{\circ}$ (north or south) and at a distance of some $7 R_{\mathrm{J}}$ from the planet which would be outside of the Io torus. Thus refraction effects in the torus should be significantly reduced.

In Fig. 7 we show the variation of $\beta$ with $L$, for assumed values of $\delta=15^{\circ}$ and $f_{\mathrm{c}}=52 \mathrm{kHz}$. In Fig. 8, we plot the values of $\beta$ calculated for the events shown in Fig. 4, again assuming values of $f_{\mathrm{c}}=52 \mathrm{kHz}$ and $L=15$, (a) for a source in the northern hemisphere of Jupiter and (b) for a source in the southern hemisphere. Figures 7 and 8 demonstrate the implication of Eq. (6), that there can be no unique value for $\beta$ that does not involve assumed values of $L$ and $f_{\mathrm{c}}$.

We have seen in Fig. 4a that, in general, LH polarization was observed when the spacecraft was at southerly jovicentric latitudes and $\mathrm{RH}$ polarization was observed when the spacecraft was at northerly jovicentric latitudes. Commensurate with this, it can be seen in Fig. $4 \mathrm{~b}$ that all of the corresponding jovimagnetic latitudes were, respectively, southerly and northerly. In other words, Ulysses was always on the same side of the jovimagnetic equator as the source at the time of the observation. It follows from Figs. 6 and 7, however, that for $\mathrm{RH}$ polarization to be observed by the spacecraft when it is at a northerly jovimagnetic latitude, the angle $\beta$ would have to be rather small if the assumption of $10 \leq L \leq 15$ is valid. Similar considerations apply to the observation of $\mathrm{LH}$ polarization when Ulysses is at a southerly jovimagnetic latitude. This is further demonstrated in Fig. 8 where all of the measured values of $\beta$ are found to be less than about $25^{\circ}$.

However, the two-dimensional geometry shown in Fig. 6 and all of the foregoing discussion assumes that the bKOM source is radiating from the face of Jupiter directed towards Ulysses. This may not be the case because 


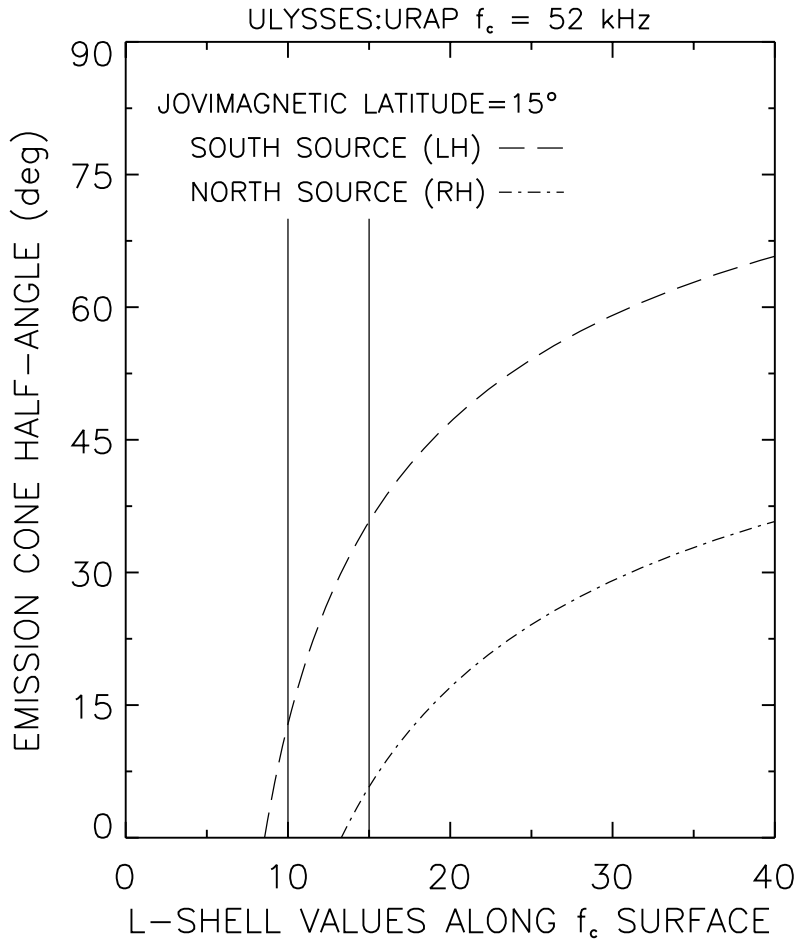

Fig. 7. Calculated values of emission cone half-angle $\beta$ against L-shell for an assumed dipole magnetic field and an emission frequency of $52 \mathrm{kHz}$

the locii of the intersections of $L$ and $f_{\mathrm{c}}$ are circles around the planet, one to the north and one to the south of the jovimagnetic equator. In the direction finding analysis presented by Ladreiter et al. (1994), it is evident that largeangle emission cones must point away from Ulysses, i.e. be close to the limb of the planet with respect to Ulysses, if their edges are to radiate in the direction of the spacecraft. This may well be the case although the following questions then arise:

1. If the cone angle is large why was $\mathrm{RH}$ polarized bKOM not received after encounter or, indeed, at any time when Ulysses was in a southerly jovicentric latitude?

2. And, similarly, why was LH polarization only received when Ulysses was in southerly jovimagnetic latitudes and RH polarization only when Ulysses was in northerly jovimagnetic latitudes?

If the cones angles are large, there should be configurations where bKOM originating in one hemisphere of Jupiter could have been received by Ulysses when the spacecraft was in the opposite hemisphere; and yet this does not seem to have occurred during the period studied here which covers almost the whole range of jovicentric latitudes occupied by the spacecraft.

Thus it may be necessary to question some of the conclusions of the CMI theory or, indeed, the suitability of the theory itself as an explanation of the bKOM emission process although, at present, there is no obvious alternative explanation available.
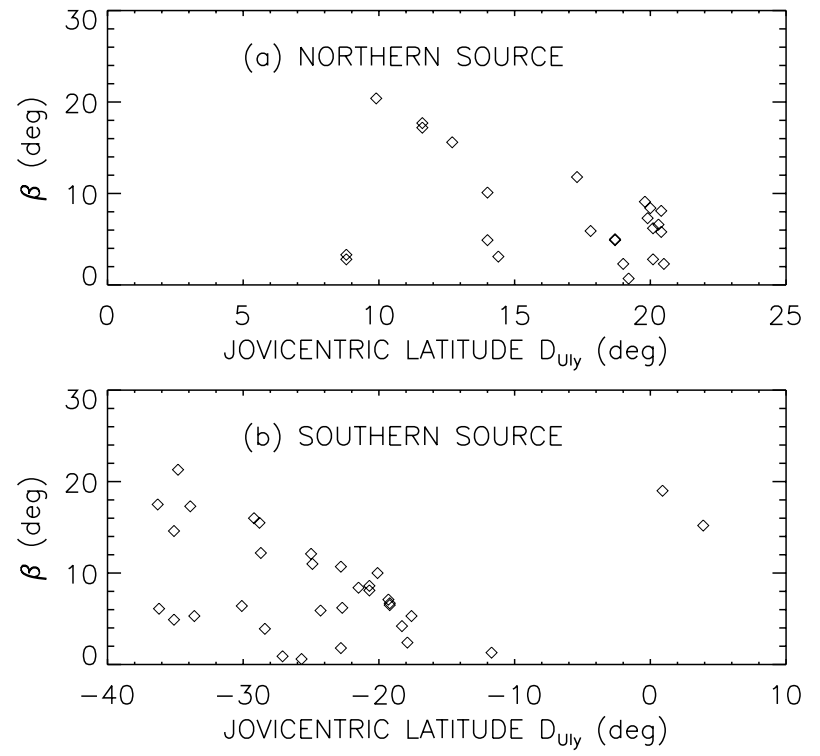

Fig. 8. Calculated values of emission cone half-angle $\beta$, for the events represented in Fig. 4, against jovicentric latitude for a) a northern jovimagnetic hemisphere source $(\mathrm{RH})$, and b) a southern jovimagnetic hemisphere source $(\mathrm{LH})$

\section{Conclusion}

We have studied the bKOM radio emission from Jupiter observed by the URAP experiment on board Ulysses during the period 940101 to 960301 , when the spacecraft was passing from south to north of the jovian equatorial plane at distances ranging from 4.7 to $7.2 \mathrm{AU}$. It is found that, in general, the bKOM events observed by Ulysses when the spacecraft was at northerly jovicentric latitudes were RH polarized while events recorded when the spacecraft was at southerly jovicentric latitudes were LH polarized, the changeover taking place somewhere between $3.9^{\circ} \leq D_{\text {Uly }} \leq 8.8^{\circ}$, close to $0^{\circ}$ jovimagnetic latitude.

Occurrence probabilities for left-hand polarized events were lower than those found previously by Barrow \& Lecacheux (1995) but similar in distribution. For the righthand polarized events, however, the distribution was different, the so-called main peak being absent or, perhaps, displaced to a higher CML.

We have shown that, in the two-dimensional model, the detection of bKOM, at a given frequency $f_{\mathrm{c}}$ by a spacecraft at a given location specified by $\delta$, determines a unique value of $\beta$ for an assumed value of $L$ and a given field model. This is not true for a three-dimensional model.

If the emission cones are assumed to be directed towards Ulysses, calculated values of $\beta$ for all of the events reported here, assuming values of $L=15$ and $f_{\mathrm{c}}=52 \mathrm{kHz}$, are found to be less than about $25^{\circ}$. Larger values of $\beta$ are possible, in principle, but then the source must be on the limb of the planet with respect to the observer. This raises difficulties concerning the polarization sense observed to either side of the jovimagnetic equatorial plane. 
It may be necessary to question some of the conclusions of the CMI theory or, indeed, the suitability of the theory itself as an explanation of the bKOM emission process although there is no obvious alternative explanation available at the present time.

Acknowledgements. We thank Drs. G. Woan and P. Zarka for valuable discussions. URAP is the collaborative effort of the four institutions, NASA Goddard Space Flight Center, Observatoire de Paris-Meudon, Centre de Recherches en Physique de l'Environnement Terrestre et Planétaire and the University of Minnesota. C.H.B. is pleased to acknowledge support from Copernicus Gesellschaft e.V. during a one-month visit to the Observatoire de Paris-Meudon.

\section{References}

Alexander, J. K., Carr, T. D., Thieman, J. R., Schauble, J. J., \& Riddle, A. C. 1981, JGR, 86, 8529

Barrow, C. H., \& Lecacheux, A. 1993, A\&A, 271, 335

Barrow, C. H., \& Lecacheux, A. 1995, A\&A, 301, 903

Barrow, C. H., Woan, G., \& MacDowall, R. J. 1999, A\&A, 344, 1001

Barrow, C. H., Lecacheux, A., MacDowall, R. J., \& Kaiser, M. L. 2001, Planet. Space Sci., in press

Boischot, A., Lecacheux, L., Kaiser, M. L., Desch, M. D., \& Alexander, J. K. 1981, JGR, 86, 8213

Boischot, A. 1988, Radio Emissions from Planetary Magnetospheres II, Proceedings of an international workshop held at Graz, Austria, ed. H. O. Rucker, S. J. Bauer, \& B. M. Pedersen (Verlag der Österreichischen Akademie der Wissenschaften), 15
Brown, L. W. 1974, ApJ, 194, L159

Carr, T. D., Desch, M. D., \& Alexander, J. K. 1983, Physics of the Jovian Magnetosphere, ed. A. J. Dessler (Cambridge University Press, Cambridge), 226

Chapman, S., \& Bartels, J. 1951, Geomagnetism, vol. I (Oxford University Press, Oxford)

Desch, M. D., \& Carr, T. D. 1974, ApJ, 194, L57

Kaiser, M. L. 1977, JGR, 82, 1256

Kaiser, M. L., \& Desch, M. D. 1984, Rev. Geophys., 22, 373

Jones, D. 1988, Radio Emissions from Planetary Magnetospheres II, Proceedings of an international workshop held at Graz, Austria, ed. H. O. Rucker, S. J. Bauer, \& B. M. Pedersen (Verlag der Österreichischen Akademie der Wissenschaften), 255

Ladreiter, H. P., Zarka, P., \& Lecacheux, A. 1994, Planet Space Sci., 42, 913

Leblanc, Y., \& Daigne, G. 1985, JGR, 90, 12073

Leblanc, Y. 1988, Radio Emissions from Planetary Magnetospheres II, Proceedings of an international workshop held at Graz, Austria, ed. H. O. Rucker, S. J. Bauer, \& B. M. Pedersen (Verlag der Österreichischen Akademie der Wissenschaften), 149

Lecacheux, A., \& Aubier, M. G. 1997, Radio Emissions from Planetary Magnetospheres IV, Proceedings of an international workshop held at Graz, Austria, ed. H. O. Rucker, S. J. Bauer, \& A. Lecacheux (Verlag der Österreichischen Akademie der Wissenschaften), 313

Manning, R., \& Fainberg, J. 1980, Space Sci. Instr., 5, 161

Stone, R. G., Bougeret, J. L., Caldwell, J., et al. 1992a, A\&AS, 92,291

Stone, R. G., Pedersen, B. M., Harvey, C. C., et al. 1992b, Science, 257, 1524

Woan, G. 1997, in ESA Report SCI-97-002, 25

Woan, G. 1999, private communication 